\title{
DENTAL RADIOGRAPHS FOR THE HOMEBOUND
}

\author{
By H. Barry Waldman, D.D.S., M.P.H. ${ }^{1}$
}

\author{
Fims exposed and radiographs developed in a home no longer \\ are impossibilities in Cleveland.
}

Portable dental engines have been available to the profession for many years. Compact "portable dental offices," with combined engine, source of light, suction, and air-pressure, permit the dentist to bring a full range of dental services to the shut-in patient, right in his home.

Unfortunately, most dental care at home is carried out without the aid of radiographs. "Portable" $\mathrm{x}$-ray units, it is true, have been available, but portable only for the extremely hardy practitioner. Weights of 75 to 100 or more pounds were not uncommon for such machines. The need for radiographs usually has been answered by bringing the patient somehow, at least once, to the private office or clinic. For the patient who just could not be moved, there has been no simple technic.

Highland View Hospital's Dental Home Care and Out Patient Program* has been providing dental service to shut-in patients now for more than five years. For the past many months, the program has been testing an extremely versatile portable x-ray unit-the Serend-20. The entire unit, (including the stand, the generator and two carrying cases), weighs but 34 pounds. It is ideally suited for use in private home or in nursing homes since it is lightweight, simple to put together for operating or for collapsing for packing and entirely safe to use." The machine was tested for safety by the Ohio State Department of Health and was found to be well within the limits and standards set forth by the National Bureau of Standards in Handbook No. 76.

The generator is suspended from a four-legged telescoping stand. The unit can be raised, lowered, swiveled, or angled to adjust to the position of the patient's head. Within a matter of five minutes, the stand can be assembled, the generator attached, an aluminum filter and columnator positioned, and the machine plugged into any common household electric outlet. A remote electronic timer permits the operator to avoid the source of radiation. Figure 1 shows the assembled equipment.

No difference in radiographic quality has been noted between the radiographs produced by the portable unit and those produced by the standard equipment in an office.

Quite frequently an immediate examination of a radiograph is necessary to permit a dental procedure at the patient's residence. A Clarus Daylight Processing Unit has been used by the Home Care Program to permit rapid developing of exposed films (Figure 2). The Clarus Unit is but 11 inches by five inches

1. Director, Dental Home Care and Out Patient Program, Highland View Hospital, now a graduate student of Medical Care Administration, School of Public Health, The University of Michigan, Ann Arbor 48104.

- Supported in part by Grant No. C. H. $27-18$ of U. S. P. H. S., Community Health Services and Facility.

* The Serend-20 unit is distributed by Winfield Inc. of Chicago, Ill., and the distributor's list-price is \$825.00. A similar unit, the Kramex PX-20, is clistributed by the Krame X Corporation of New York City and the distributor's list-price is $\$ 975.00$. 


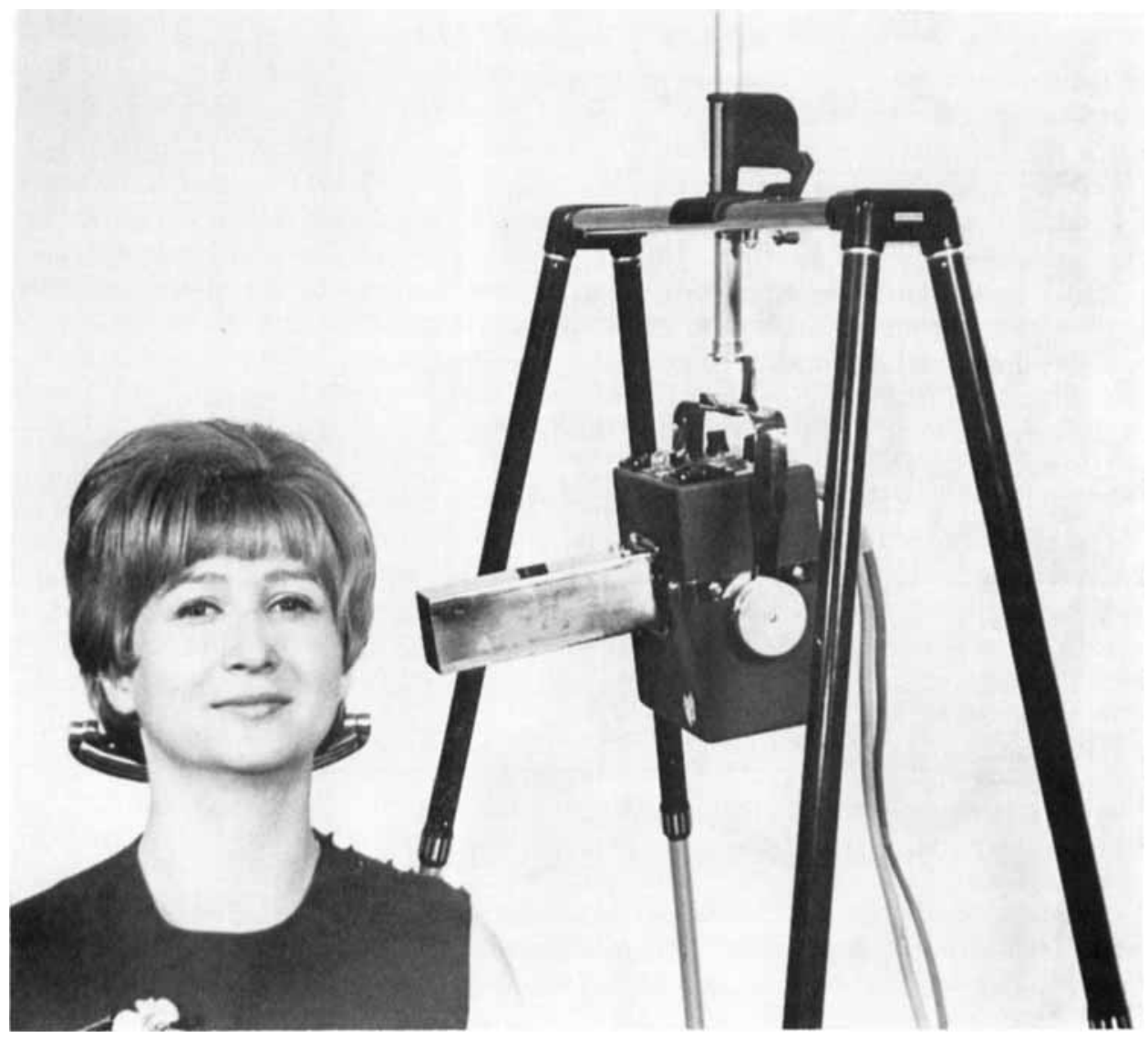

Figure 1. Assembled Radiographic Equipment

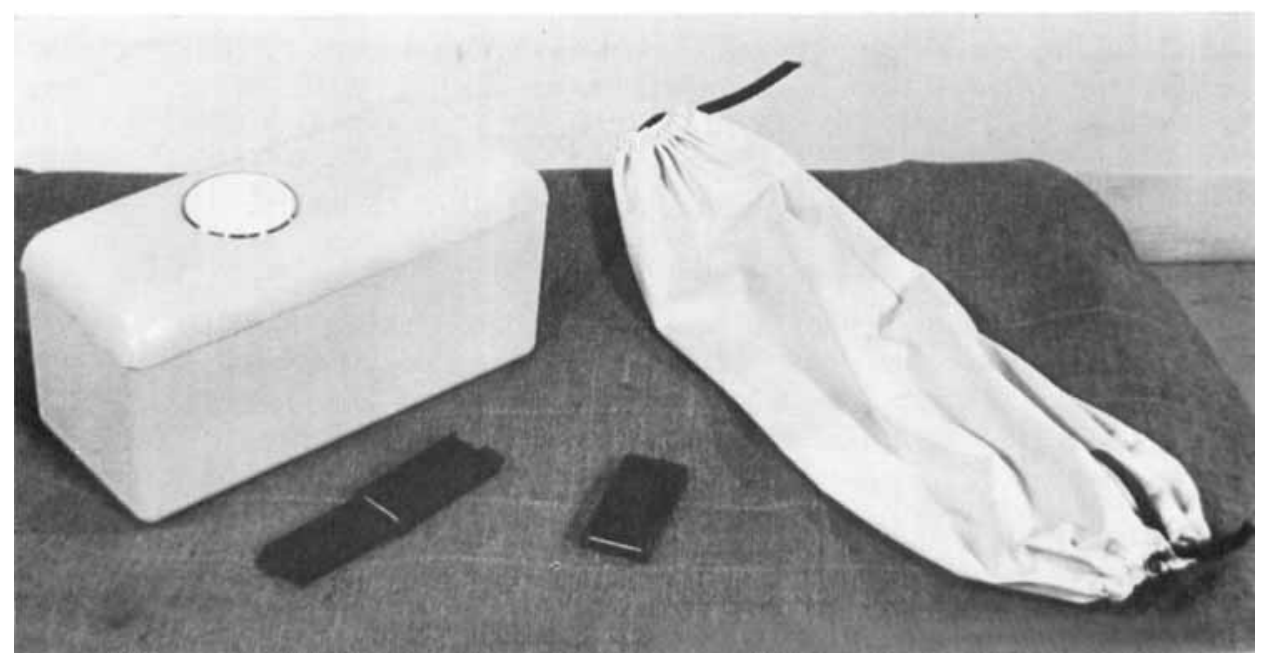

Figure 2. Clarus Daylight Processing Unit 
by six inches, and it weighs less than three pounds. Its unusual compactness and negligible weight make it an ideal companion for the portable x-ray unit.

The developing unit consists of a series of four tanks and a light-proof bag for placing the exposed film into daylight cassettes of transparent red plastic. Once the film has been transferred into the cassette, it is removed from the lightproof bag and placed in the developing tank. The unit then is covered, the timer on top of the unit is set, and the film is permitted to remain in the developer for the prescribed period of time. Subsequently, the film is washed, fixed, then washed again, and permitted to dry. The film remains in the plastic cassette throughout the entire procedure of developing and fixing and only is removed for the final washing and drying. After the completion of the procedure, the developer and fixer solutions can be returned to the carrying bottles. The Home Care team then is ready to perform the needed dental procedure after a satisfactory diagnosis.

Again, it can be shown that no difference in quality can be noted between those radiographs developed in the portable developing unit and those processed in the customary dark room of an office. The portable $x$-ray machine and developing units have become, hence, important additions to the equipment of the Unit for Home Dental Care, and the operators are pleased that they no longer must perform dental services in a home without the diagnostic aid of radiographs.

\section{Rinsing and Toothbrushing with Sodium Fluoride}

Berggren of Stockholm, Sweden, reports that supervised toothbrushing of school children at intervals of two months with 0.5 percent solution of sodium fluoride has resulted in a considerable decrease in the rate of caries-attack of the teeth of older children and the prevalence of periodontal disease. (International Dental Journal for March, 1967) 